\title{
Noise Model Analysis of MEMS Gyroscope Circuit Control System
}

\author{
Yuxian $\mathrm{Liu}^{1}$, Ting Chen ${ }^{1}$ and Chunhua $\mathrm{He}^{2, *}$ \\ ${ }^{1}$ GuangDong ShunDe Innovation Design Institute, FoShan 528311, China \\ ${ }^{2}$ National Key Laboratory of Science and Technology on Micro/Nano Fabrication Institute of Microelectronics, Beijing 100871, \\ China \\ ${ }^{*}$ Corresponding author
}

\begin{abstract}
The detection signal of MEMS resonant gyroscope is very weak, and it is sensitively affected by the mechanical structure and the noise of the detection circuit. Noise is an important parameter which affecting gyroscope resolution, angle random walk coefficient and zero partial stability. In this paper, the noise model is established for the front-end solitary circuit, filter circuit, ADC/DAC and other key circuit modules of the gyro control system drive closed loop and sense closed loop, through the mathematical theory, the paper deduces the noise of the key circuit module, instructs the control system parameter design, and gives the corresponding simulation results.
\end{abstract}

Keywords-MEMS gyroscope; noise model; front-end circuit; filter; $A D C / D A C$

\section{INTRODUCTION}

MEMS gyroscope has advantages of small size, light weight, low price, long life and easy to mass production, it is widely used in consumer electronics, automotive safety system, robot and inertial navigation field ${ }^{[1]}$. The detection signal of MEMS gyro is very weak, and the control detection circuit system of high signal-to-noise ratio is very important to improve its performance. Gyroscope noise mainly includes mechanical thermal noise and circuit noise. The model of mechanical thermal noise ${ }^{[2-6]}$ is relatively simple and the research of mechanical thermal noise relatively mature. It can be suppressed by optimizing the structural design parameters and vacuum packaging. In contrast, the circuit noise is relatively complicated ${ }^{[7-10]}$, the noise source mainly comes from the noise introduced by the circuit and each module of the system, such as RC noise, amplifier, multiplier, quantization noise of ADC / DAC, cut off of digital circuit noise and so on. In addition, the noise of the circuit is much larger than the mechanical thermal noise, which plays a major role in the total noise, therefore, this paper analyzes the noise model of the front-end readout circuit, op amp circuit and ADC / DAC circuit module in detail, in order to guide the parameter design of the circuit system, the corresponding mathematical theory is deduced and the corresponding experimental data are given.

\section{Front-END READOUT CiRCUIT NOISE ANALYSIS}

The front-end readout circuit with feed-forward coupling compensation is shown in Figure 1, where $V_{n} O_{p 1}$ and $V_{n} O_{p 2}$ are the input power noise power spectral density of the operational amplifier, $I_{n_{-} O n 1}$ and $I_{n_{-} O n 2}$ are the input current noise power spectral density of the negative terminal of the operational amplifier, $V_{n \_D a}$ is the input voltage noise power spectral density of the instrument amplifier , $I_{n \text { Dap }}$ and $I_{n \text { Dan }}$ are respectively the input current noise power spectral density of The positive and negative instrumentation amplifier, $V_{n_{-} R g}$, $V_{n R f 1}, V_{n R f 2}, V_{n R h 1}$ and $V_{n R h 2}$ are respectively the noise voltages of the resistors $R_{g}, R_{f 1}, R_{f 2}, R_{h 1}$ and $R_{h 2}$, Johnson noise can be obtained:

$$
\begin{gathered}
\frac{V_{n_{-} R g}}{\sqrt{\Delta f}}=\sqrt{4 k_{b} T R_{g}}, \frac{V_{n_{-} R f 1}}{\sqrt{\Delta f}}=\sqrt{4 k_{b} T R_{f 1}}, \\
\frac{V_{n_{-} R f 2}}{\sqrt{\Delta f}}=\sqrt{4 k_{b} T R_{f 2}}, \frac{V_{n_{-} R h 1}}{\sqrt{\Delta f}}=\sqrt{4 k_{b} T R_{h 1}}, \\
\frac{V_{n_{-} R h 2}}{\sqrt{\Delta f}}=\sqrt{4 k_{b} T R_{h 2}}
\end{gathered}
$$

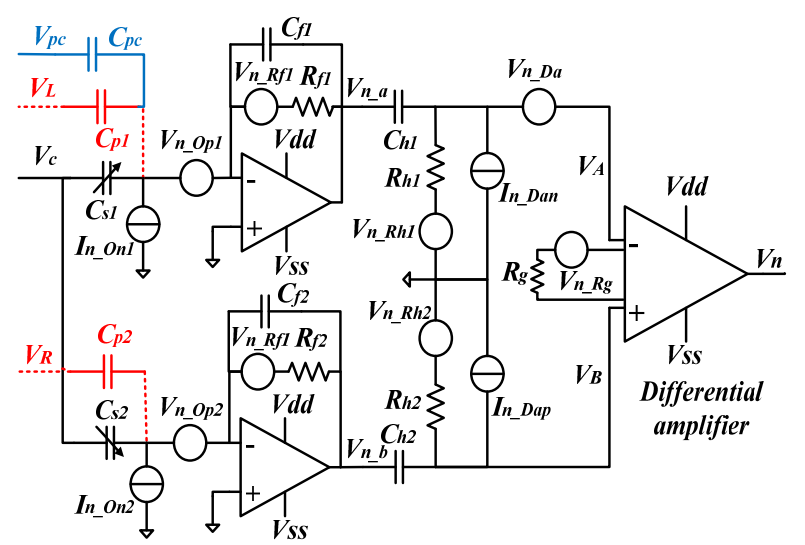

FIGURE I. FRONT-END READOUT CIRCUIT NOISE ANALYSIS

The output voltage noise power spectral density of the two op amps is respectively:

$$
\begin{aligned}
& \frac{V_{n a}^{2}}{\Delta f}=\frac{4 k_{b} T R_{f 1}}{1+\omega^{2} R_{f 1}^{2} C_{f 1}^{2}}+\frac{I_{n_{-} o n 1}^{2} R_{f 1}^{2}}{1+\omega^{2} R_{f 1}^{2} C_{f 1}^{2}} \\
& +\frac{V_{n_{-} o p 1}^{2}\left[1+\omega^{2} R_{f 1}^{2}\left(C_{f 1}+C_{s 1}+C_{p 1}+C_{p c}\right)^{2}\right]}{1+\omega^{2} R_{f 1}^{2} C_{f 1}^{2}}
\end{aligned}
$$




$$
\begin{aligned}
& \frac{V_{n b}^{2}}{\Delta f}=\frac{4 k_{b} T R_{f 2}}{1+\omega^{2} R_{f 2}^{2} C_{f 2}^{2}}+\frac{I_{n_{-} O n 2}^{2} R_{f 2}^{2}}{1+\omega^{2} R_{f 2}^{2} C_{f 2}^{2}} \\
& +\frac{V_{n_{-} O p 2}^{2}\left[1+\omega^{2} R_{f 2}^{2}\left(C_{f 2}+C_{s 2}+C_{p 2}\right)^{2}\right]}{1+\omega^{2} R_{f 2}^{2} C_{f 2}^{2}}
\end{aligned}
$$

Since the noise of the two op amps is uncorrelated with each other, the output voltage noise power spectral density of the instrument amplifier is the sum of the individual noise:

$$
\begin{aligned}
& \frac{V_{n}^{2}}{\Delta f}=k_{g}^{2}\left[\frac{V_{n a}^{2}}{\Delta f} \frac{\omega^{2} R_{h 1}^{2} C_{h 1}^{2}}{1+\omega^{2} R_{h 1}^{2} C_{h 1}^{2}}+\frac{V_{n b}^{2}}{\Delta f} \frac{\omega^{2} R_{h 2}^{2} C_{h 2}^{2}}{1+\omega^{2} R_{h 2}^{2} C_{h 2}^{2}}\right. \\
& +\frac{4 k_{b} T R_{h 1}}{1+\omega^{2} R_{h 1}^{2} C_{h 1}^{2}}+\frac{4 k_{b} T R_{h 2}}{1+\omega^{2} R_{h 2}^{2} C_{h 2}^{2}}+\frac{I_{n_{-} D a n}^{2} R_{h 1}^{2}}{1+\omega^{2} R_{h 1}^{2} C_{h 1}^{2}} \\
& \left.+\frac{I_{n_{-} D a p}^{2} R_{h 2}^{2}}{1+\omega^{2} R_{h 2}^{2} C_{h 2}^{2}}+V_{n_{-} D a}^{2}+4 k_{b} T R_{g}\right]
\end{aligned}
$$

Among them, the gain of the instrument amplifier is $k_{g}$. The following mainly consider the noise of the gyro-driven resonance frequency $\omega_{d}$, assuming that $\Delta C_{s 1}=\Delta C_{s 2}=\Delta C_{s}, C_{s 01}$ $=C_{s 02}=C_{0}, C_{f 1}=C_{f 2}=C_{f}, R_{f 1}=R_{f 2}=R_{f}, C_{p 1}=C_{p 2}=C_{p}, C_{h 1}$ $=C_{h 2}=C_{h}, R_{h 1}=R_{h 2}=R_{h}, 1>>\omega_{d} C_{f} R_{f}, 1<<\omega_{d} C_{h} R_{h}, I_{n_{-} D a p}$ $=I_{n_{-} D a n}=I_{n_{-} D a}, V_{n_{-} O p 1}=V_{n_{-} O p 2}=V_{n_{-} O p}, I_{n_{-} O n 1}=I_{n_{-} O n 2}=I_{n_{-} O n}$, Then the above formula simplifies:

$$
\begin{gathered}
\frac{V_{n a}^{2}}{\Delta f} \approx 4 k_{b} T R_{f 1}+I_{n_{-} O n 1}^{2} R_{f 1}^{2}+V_{n_{-} O p 1}^{2} \\
=4 k_{b} T R_{f}+I_{n_{-} O n}^{2} R_{f}^{2}+V_{n_{-} O p}^{2} \\
\frac{V_{n b}^{2}}{\Delta f} \approx 4 k_{b} T R_{f 2}+I_{n_{-} O n 2}^{2} R_{f 2}^{2}+V_{n_{-} O p 2}^{2} \\
=4 k_{b} T R_{f}+I_{n_{-} O n}^{2} R_{f}^{2}+V_{n_{-} O p}^{2} \\
\frac{V_{n}}{\sqrt{\Delta f}} \approx k_{g} \times\left[2 \times\left(4 k_{b} T R_{f}+I_{n_{-} O n}^{2} R_{f}^{2}\right.\right. \\
\left.\left.+V_{n_{-} O p}^{2}+\frac{4 k_{b} T}{\omega_{d}^{2} R_{h} C_{h}^{2}}+\frac{I_{n_{-} D a}^{2}}{\omega_{d}^{2} C_{h}^{2}}\right)+V_{n_{-} D a}^{2}+4 k_{b} T R_{g}\right]^{1 / 2}
\end{gathered}
$$

It can be seen that in order to suppress the noise, high bandwidth, high slew rate, low noise precision operational amplifier and instrumentation amplifier should be selected, so that the input voltage noise power spectral density and input current noise power spectral density as low as possible. In addition, $\omega_{d}, C_{h}, R_{h}$ can be appropriately increased to reduce noise, And increasing $\omega_{d}, C_{h}, R_{h}$ by decreasing $k_{g}, R_{g}, R_{f}$ is easier to achieve as long as $1<<\omega_{d} C_{h} R_{h}$ is satisfied. The inverse relationship between $R_{g}$ and $k_{g}$ can not therefore

increase or decrease at the same time. If $k_{g}$ and $R_{f}$ are reduced, then the gain of the useful signal will also be reduced.

\section{OP AMP CIRCUIT NOISE ANALYSIS}

The op amp circuit of gyro circuit control system includes inverter, high-pass filter and low-pass filter. This part mainly analyzes the noise model of these circuits.

\section{A. Inverter Noise Analysis}

The noise original circuit and noise analysis of the inverting amplifier are shown in Figure 2 (a) and (b), The expressions for gain and output noise are based on this circuit:

$$
\begin{gathered}
\frac{V_{\text {out }}}{V_{i}}=\frac{-R_{f}}{R_{i}} \\
\frac{V_{n}^{2}}{\Delta f}=4 k_{b} T R_{f}+\left(1+R_{f} / R_{i}\right)^{2}\left(4 k_{b} T R_{c}+I_{n_{-} O p}^{2} R_{c}^{2}+V_{n_{-} O p}^{2}\right) \\
+4 k_{b} T R_{i}\left(R_{f} / R_{i}\right)^{2}+I_{n_{-} O n}^{2} R_{f}^{2}
\end{gathered}
$$



(a)

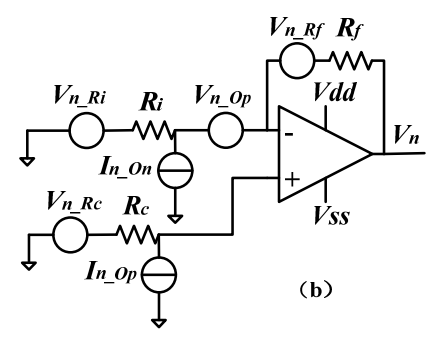

FIGURE II. INVERTING AMPLIFIER CIRCUIT NOISE ANALYSIS

Among them, $R_{c}$ is a matching resistor, since the op amp inevitably has input offset current (that is, the error of the bias current of the two differential inputs). therefore, the design of high-precision DC amplifier, in order to balance the input bias current, it is necessary to equalize the DC path resistance across the op amp, that is, $R_{c}=R_{i} / / R_{f}=R_{i} R_{f} /\left(R_{i}+R_{f}\right)$. However, for a high-precision AC amplifier, the amount of direct current is not important, and the mismatch caused by the bias current can be filtered out in the subsequent high-pass filter and angular velocity demodulation. So here set $R_{c}=0$, so that the noise caused by this item to a minimum, at this point, the signal-to-noise ratio can be expressed as:

$$
\begin{aligned}
& S N R=\frac{V_{\text {out }}}{V_{n}} \\
& =\frac{V_{i}}{\left[4 k_{b} T\left(R_{i}+R_{i}^{2} / R_{f}\right)+\left(1+R_{i} / R_{f}\right)^{2} V_{n_{-} O p}^{2}\right.} \\
& \left.\quad+R_{i}^{2} I_{n_{-} O n}^{2}\right]^{1 / 2}
\end{aligned}
$$


According to the expression of signal-to-noise ratio, we can see that in order to reduce the influence of noise, besides selecting high-precision operational amplifier to make $I_{n}$ On, $I_{n} O p$ and $V_{n_{O} O p}$ as low as possible, $R_{i}$ and $R_{i} / R_{f}$ should be minimized. If $R_{i} / R_{f}$ is reduced, the circuit gain increases, but the power consumption of the circuit will also increase, so the choice of resistance should be properly compromised. Under different design resistances, the experimental results of the output noise of the inverting amplifier are shown in Table 1. When $R_{f}$ and $R_{i}$ are constant, the smaller the $R_{c}$ is, the smaller the noise is. When $R_{i} / R_{f}$ and $R_{c}$ are not changed, the smaller the $R_{i}$ is, the smaller the noise is. This shows that the experimental test results consistent with the theoretical analysis.

TABLE I. INVERTED AMPLIFIER OUTPUT NOISE EXPERIMENTAL TEST RESULTS

\begin{tabular}{|c|c|c|c|c|}
\hline Test times & $\boldsymbol{R i}(\boldsymbol{\Omega})$ & $\boldsymbol{R} \boldsymbol{f}(\boldsymbol{\Omega})$ & $\boldsymbol{R} \boldsymbol{(}(\boldsymbol{\Omega})$ & $\boldsymbol{V n}(\boldsymbol{\mu} \boldsymbol{)})$ \\
\hline 1 & $1 \mathrm{k}$ & $1 \mathrm{k}$ & 0 & 0.096 \\
\hline 2 & $1 \mathrm{k}$ & $1 \mathrm{k}$ & $10 \mathrm{M}$ & 3 \\
\hline 3 & $10 \mathrm{k}$ & $10 \mathrm{k}$ & 0 & 0.1 \\
\hline 4 & $10 \mathrm{k}$ & $10 \mathrm{k}$ & $10 \mathrm{k}$ & 0.11 \\
\hline 5 & $100 \mathrm{k}$ & $100 \mathrm{k}$ & 0 & 0.13 \\
\hline 6 & $100 \mathrm{k}$ & $100 \mathrm{k}$ & $100 \mathrm{k}$ & 0.19 \\
\hline 7 & $1 \mathrm{M}$ & $1 \mathrm{M}$ & 0 & 0.35 \\
\hline 8 & $1 \mathrm{M}$ & $1 \mathrm{M}$ & $1 \mathrm{M}$ & 0.59 \\
\hline 9 & $10 \mathrm{M}$ & $10 \mathrm{M}$ & 0 & 1.98 \\
\hline 10 & $10 \mathrm{M}$ & $10 \mathrm{M}$ & $10 \mathrm{M}$ & 2.73 \\
\hline
\end{tabular}

\section{B. Filter Noise Analysis}

The circuit noise analysis diagram of first-order low-pass filter and first-order high-pass filter is shown in figure 3. For a first-order low-pass filter, the transfer function and noise are as follows:

$$
\begin{gathered}
\frac{V_{\text {out }}}{V_{i}}=\frac{-R_{f} / R_{i}}{1+s R_{f} C_{f}} \\
\frac{V_{n}^{2}}{\Delta f}=\frac{4 k_{b} T R_{f}}{1+\omega^{2} R_{f}^{2} C_{f}^{2}}+\frac{\left(1+R_{f} / R_{i}\right)^{2}+\omega^{2} R_{f}^{2} C_{f}^{2}}{1+\omega^{2} R_{f}^{2} C_{f}^{2}} \\
\left(4 k_{b} T R_{c}+I_{n_{-} O p}^{2} R_{c}^{2}+V_{n_{-} O p}^{2}\right)+\frac{4 k_{b} T R_{i}\left(R_{f} / R_{i}\right)^{2}}{1+\omega^{2} R_{f}^{2} C_{f}^{2}} \\
+\frac{I_{n_{-} O n}^{2} R_{f}^{2}}{1+\omega^{2} R_{f}^{2} C_{f}^{2}}
\end{gathered}
$$

In order to suppress the noise, let $\mathrm{Rc}_{\mathrm{c}}=0$. Considering the noise in the passband, there is $1>>\omega C_{f} R_{f}$, so that the simplified signal to noise ratio is obtained:

$$
\begin{aligned}
& S N R=\frac{V_{\text {out }}}{V_{n}} \\
& \approx \frac{V_{i}}{\left[4 k_{b} T\left(R_{i}+R_{i}^{2} / R_{f}\right)+\left(1+R_{i} / R_{f}\right)^{2} V_{n_{-} O p}^{2}\right.} \\
& \left.\quad+R_{i}^{2} I_{n_{-} O n}^{2}\right]^{1 / 2}
\end{aligned}
$$
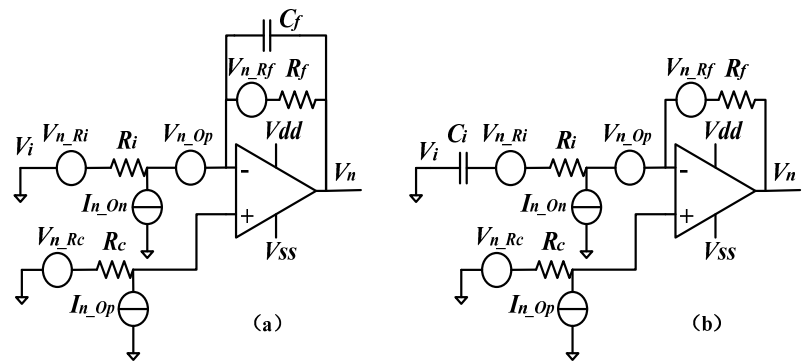

FIGURE III. CIRCUIT NOISE ANALYSIS OF FIRST-ORDER LOW PASS FILTER AND FIRST-ORDER HIGH-PASS FILTER.

Similarly, according to the derivation of the first-order lowpass filter, when $1<<\omega C_{f} R_{f}$, the signal-to-noise ratio of the first-order high-pass filter can be obtained as:

$$
\begin{aligned}
& S N R=\frac{V_{\text {out }}}{V_{n}} \\
& \approx \frac{V_{i}}{\left[4 k_{b} T\left(R_{i}+R_{i}^{2} / R_{f}\right)+\left(1+R_{i} / R_{f}\right)^{2} V_{n_{-} O p}^{2}\right.} \\
& \left.\quad+R_{i}^{2} I_{n_{-} O n}^{2}\right]^{1 / 2}
\end{aligned}
$$

\section{ADC / DAC CIRCUIT NOISE ANALYSIS}

This part analyzes the noise of ADCs and DACs for digital circuits, taking the sense closed-loop system as an example to be derived, the block diagram of the sense closed-loop noise analysis system is shown in Fig. $4^{[11]}$, where $V_{n_{-} A D C}$ is the noise voltage brought by $\mathrm{ADC}, V_{n_{-} D A C}$ is the noise voltage brought by DAC, $V_{f}$ is the feedback voltage, Because $V_{f}$ is the closest to the angular velocity output signal in the system, it is necessary to minimize the noise voltage of $V_{f}$ to reduce the angular velocity of the noise voltage.

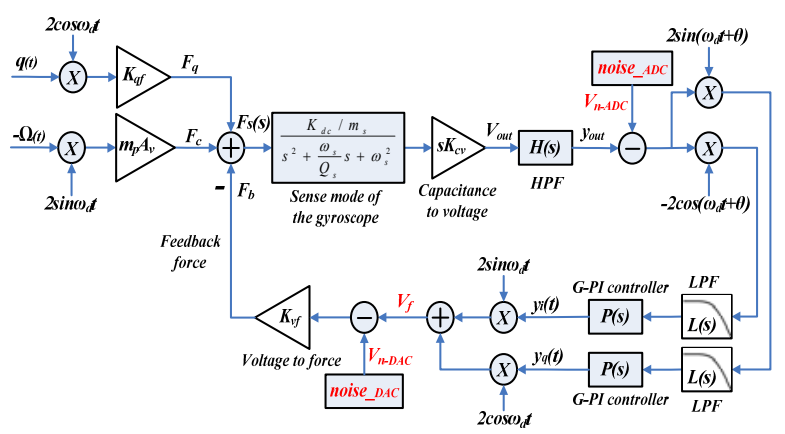

FIGURE IV. BLOCK DIAGRAM OF SENSE CLOSED-LOOP ADC AND DAC NOISE ANALYSIS SYSTEM. 
Laplace changes in the control link are as follows:

$$
\begin{gathered}
H(s)=\frac{s}{s+\omega_{h}} \\
L(s)=\frac{\omega_{l}^{2}}{s^{2}+2 \xi_{l} \omega_{l} s+\omega_{l}^{2}} \\
P(s)=\frac{k_{p}\left(s+\omega_{2}\right)}{\left(s+\omega_{1}\right)\left(s+\omega_{3}\right)}
\end{gathered}
$$

So the transfer function of ADC and DAC noise is:

$$
\begin{gathered}
T N_{A D C}(s)=\frac{V_{f}}{V_{n_{-} A D C}} \\
{\left[2 j \left[e^{-j \theta} L\left(s-j \omega_{d}\right) P\left(s-j \omega_{d}\right)\right.\right.} \\
=\frac{\left.\left.-e^{j \theta} L\left(s+j \omega_{d}\right) P\left(s+j \omega_{d}\right)\right]\right]}{\left[1+2 j s k_{c v} k_{v f}\left[e^{-j \theta} L\left(s-j \omega_{d}\right) P\left(s-j \omega_{d}\right)\right.\right.} \\
\left.\left.-e^{j \theta} L\left(s+j \omega_{d}\right) P\left(s+j \omega_{d}\right)\right] H(s) G_{s}(s)\right] \\
T N_{D A C}(s)=\frac{V_{f}}{V_{n_{-} D A C}} \\
{\left[2 j s k _ { c v } k _ { v f } \left[e^{-j \theta} L\left(s-j \omega_{d}\right) P\left(s-j \omega_{d}\right)\right.\right.} \\
\left.\left.-e^{j \theta} L\left(s+j \omega_{d}\right) P\left(s+j \omega_{d}\right)\right] H(s) G_{s}(s)\right] \\
{\left[1+2 j s k_{c v} k_{v f}\left[e^{-j \theta} L\left(s-j \omega_{d}\right) P\left(s-j \omega_{d}\right)\right.\right.} \\
\left.\left.-e^{j \theta} L\left(s+j \omega_{d}\right) P\left(s+j \omega_{d}\right)\right] H(s) G_{s}(s)\right]
\end{gathered}
$$

According to the above formula, the frequency response curve of $T N_{A D C}(s)$ and $T N_{D A C}(s)$ can be simulated, as shown in Fig.5.It can be seen that the noise of the DAC is more affected than the noise of the ADC. Assuming that the input noise of $\mathrm{ADC}$ and DAC is white noise, as shown in Fig. 6, Simulink software based on Matlab can simulate the system in time domain. The results are shown in Fig. 7 and Fig. 8. It can be seen that the same input white noise, the output noise angular velocity caused by DAC noise is greater than the output noise caused by ADC noise, which is consistent with the conclusion of frequency domain analysis.
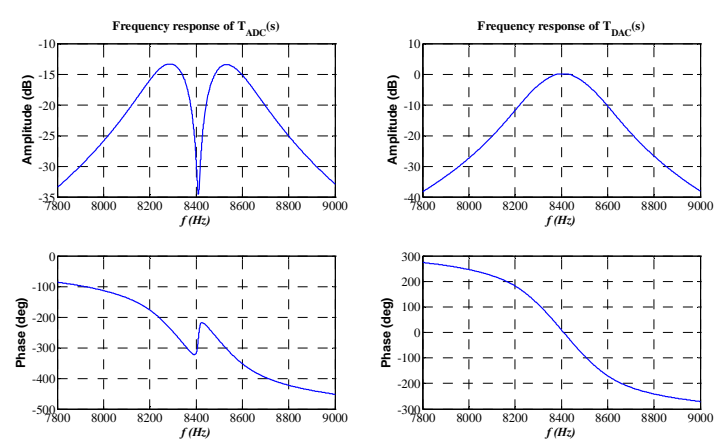

FIGURE V. TNADC(S) AND TNDAC(S) FREQUENCY RESPONSE CURVE.

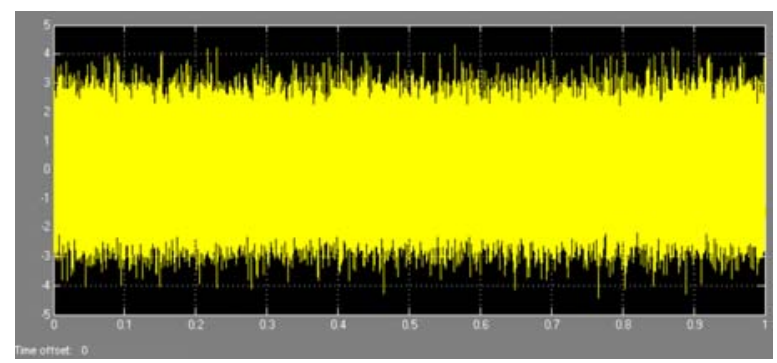

FIGURE VI. INPUT NOISE CURVES OF ADC AND DAC.

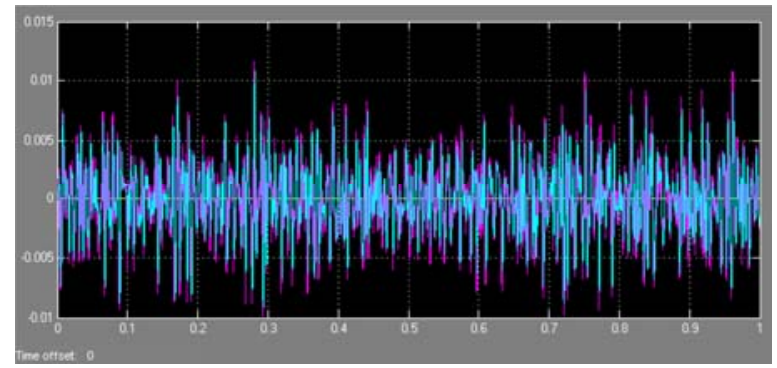

FIGURE VII. THE CHANGE CURVE OF OUTPUT ANGULAR VELOCITY CAUSED BY ADC NOISE.

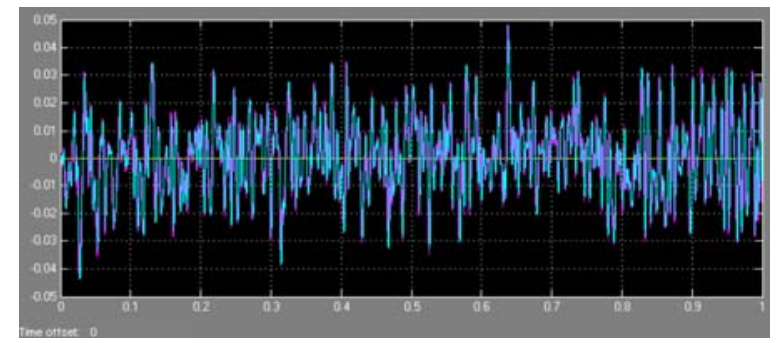

FIGURE VIII. THE CHANGE CURVE OF OUTPUT ANGULAR VELOCITY CAUSED BY DAC NOISE.

\section{CONCLUSION}

In this paper, the noise model of drive closed loop and sense closed loop links which include front-end solitary circuit, filter circuit, ADC/DAC circuit for the gyroscope control system are established to analysis the signal to noise ratio of the system. Then deduces signal to noise ratio mathematical expression of each links of the gyroscope control system, through the mathematical theory analysis which instructs the 
control system parameter design, gives the corresponding simulation results and experimental results.

\section{ACKNOWLEDGMENT}

The authors are grateful to the technical staffs of National Key Laboratory of Science and Technology on Micro/Nano Fabrication for their supports on MEMS gyroscopes fabrications.

\section{REFERENCES}

[1] X.H Zhao, G.X Yin, C.H Wang. Research on temperature compensation of silicon gyro's drift $[\mathrm{J}]$ Transducer and Microsystem Technologies, 2008, 27( 10): $48-50$.

[2] M.H. Bao, "Micro mechanical transducers: pressure sensors, accelerometers, and gyroscopes”, Amsterdam New York: Elsevier, 2000.

[3] V. Annovazzi-Lodi, S. Merlo, "Mechanical-thermal noise in micromachined gyros”, Microelectronics J., Vol. 30, 1999, pp. 12271230.

[4] R.P. Leland "Mechanical-Thermal Noise in MEMS Gyroscopes”, IEEE SENSORS J., Vol. 5, No. 3, 2005, pp. 493-500.

[5] S.A.M. Lajimi, G.R. Heppler, E.M. Abdel-Rahman, "A mechanicalthermal noise analysis of a nonlinear microgyroscope”, Mechanical Systems and Signal Processing, Vol. 83, 2017, pp. 163-175.

[6] Y. Yorozu, M. Hirano, K. Oka, and Y. Tagawa, "Electron spectroscopy studies on magneto-optical media and plastic substrate interface,” IEEE Transl. J. Magn. Japan, vol. 2, pp.

[7] E. Tatar, "Quadrature error compensation and its effects in the performance of fully decoupled MEMS gyroscope”, MS. D., Middle East Technical University, Turkey, 2010.

[8] D. Kim and R.T. M'Closkey, "Spectral Analysis of Vibratory Gyro Noise”, IEEE SENSORS J., Vol. 13, No. 11, 2013, pp. 4361-4374.

[9] H.L. Cao, H.S. Li, J. Liu, et al., "An improved interface and noise analysis of a turning fork microgyroscope structure”, Mechanical Systems and Signal Processing, Vol. 70-71, 2016, pp. 1209-1220.

[10] F. Giacci, S. Dellea, G. Langfelder, "Capacitive vs Piezoresistive MEMS Gyroscopes: A Theoretical and Experimental Noise Comparison”, Procedia Engineering, Vol. 120, 2015, pp. 406- 409.

[11] He Chunhua, "Research on Detection and Automatic Control System of Micromachined Vibratory Gyroscopes” [D], Master thesis, Peking University, 2013. 